\title{
Modelling Tumor Growth Under Angiogenesis In- hibition with Mixed-effects Models
}

\author{
Tamás Ferenci, Johanna Sápi, Levente Kovács
}

Research and Innovation Center of Óbuda University, Physiological Controls Group Óbuda University, Kiscelli utca 82, Budapest, H-1032, Hungary

Email: \{ferenci.tamas,sapi.johanna,kovacs.levente\}@nik.uni-obuda.hu

\begin{abstract}
Angiogenesis inhibitors offer a promising new treatment modality in oncology. However, the optimal administration regimen is often not well-established, despite the fact that it might have substantial impact on the outcome. The aim of the present study was to investigate this issue. Eight weeks old male C57Bl/6 mice were implanted with C38 colon adenocarcinoma, and were given either daily $(n=9)$ or single $(n=5)$ dose of bevacizumab. Outcome was measured by tracking tumor volume; both caliper and magnetic resonance imaging was employed. Longitudinal growth curves were modelled with mixed-effects models (with correction for autocorrelation and heteroscedasticity, where necessary) to infer on population-level. Several different growth models (exponential, logistic, Gompertz) were applied and compared. Results show that the estimation of the exponential model is very reliable, but it prevents extrapolation in time. Nevertheless, it clearly established the advantage of the continuous regime.
\end{abstract}

Keywords: mixed effects models; tumor growth; angiogenesis inhibition; dosing regimen

\section{Introduction}

Biomedical experiments aim to investigate and understand physiological and pathophysiological processes. There are three main types of the biomedical experiments: in vivo, in vitro and in silico experiments. In vivo studies use living organisms for the experiment, these are animal studies and clinical trials. In vitro studies examine biological processes in a controlled environment but outside of a living organism. Finally, in silico studies are performed on computer or via computer simulation. It is clear that in vivo experiments describes most precisely the real processes and as a result, we can observe the overall effects of an experiment on a living organism. The drawback, however, is the use and sacrifice of animals (in case of animal experiments), and according to the principles of the 3Rs (replacement, reduction and refinement) [1] we should avoid or replace the use of animals (replacement), and minimize the number of animals used per experiment (reduction). 
In our experiment, we used 14 mice in total (in order to fulfill the principle of reduction), nevertheless the obtained results are satisfactory to carry out statistical modelling (which is a replacement method since in the future these models can be used for further investigation instead of the use of mice).

In cancer treatment, a novel approach is identifying cancer-specific mechanisms and develop therapies based on these key points or targets. These targeted molecular therapies [2] contain several different specific treatment types. Knowing that angiogenesis (namely the formation of new blood vessels) has a key role in tumor growth, inhibiting angiogenesis could lead to slowed tumor growth or, in particular, it can cease the whole growth process [3]. Hahnfeldt et al [4] carried out a tumor growth model which describes the growth process under antiangiogenic therapy; however, the biological fundamentals of this model have become outdated.

In particular, the aim of our study was to determine whether the continuous administration of an antiangiogenic inhibitor offers advantages over the more conservative (higher dose - less frequent administration) approach.

\subsection{Tumor Growth Models}

While tumor growth involves many complicated biological mechanisms, its overall nature - in terms of weight, size or volume - often follows surprisingly simple patterns. This was recognized decades ago (especially following the landmark paper of Laird in 1964 [5]) and has been utilized - in spite of their limitations - ever since both to understand the biological foundations and to provide modelling, for instance in preclinical studies of drug candidates using xenograft tumors implanted in test animals.

These models might be purely empirical, like the Gompertzian growth discussed by Laird, or they might involve considerations about the underlying biological mechanisms (mechanistic and semi-mechanistic models), like the exponential-linear model by Simeoni [6].

Empirical models will be used in the present study. While some models (e.g. [7]) directly incorporate the effect of drugs (making them at least semi-mechanistic), now the same - empirical - growth model is assumed to apply in both the control and the treated groups; the drug exerts its effects by altering the parameters of the curve.

\subsubsection{Exponential Growth (Inital Phase, No Plateau)}

One of the earliest observations about tumor growth modeling was that in many cases, the growth - both in vivo and in vitro - exhibits exponential nature in its earliest period. Biologically, it correspends to the phase where the resource-limitation is not apparent, and in that sense the tumor can "freely" grow, limited only by its own size which defines the pool of cells that can divide. 
The growth thus obeys the following ordinary differential equation (DE):

$\frac{\mathrm{d} V(t)}{\mathrm{d} t}=a V(t)$

the solution of which is the well-known exponential growth formula:

$V(t)=V_{0} e^{a t}$,

where $V(t)$ is the tumor volume (or any dimension of the tumor, in the general case), with $V_{0}=V(0)$.

In almost every practical case, this model can only describe the early phase of tumor growth. In particular, it always leads to an infinite growth, the reason being that the effect of the appearence of growth-limiting factors is not accounted for.

As measurements in the present study were made in such initial period, apparently even the exponential growth provides adequate fit. However, these models are still problematic, even in this case, simply because they offer no possibility to extrapolate in time. Even if the plateau is not yet apparent, we might try to model it (or it might be an especially important task in such case) using only pre-plateau information this is not possible with the exponential model.

\subsubsection{Sigmoid Growth (Plateau Accounted For)}

The typical solution to this problem is the application of sigmoid-like growth curves. Such models can capture the asymptotical phase, and thus the plateau can be estimated - even from data collected before reaching the plateau. The question will be, of course, the reliability if the observations are far from the plateau-phase.

We will use two popular sigmoid models in the present study, for a review of the alternatives, see $[8,9,10]$.

Note that many such model is a special case of the DE

$\frac{\mathrm{d} V(t)}{\mathrm{d} t}=a V(t)^{\alpha}-b V(t)^{\beta}$,

which is usually called the generalized two-parameter model, with appropriate choice of the $a, \alpha, b, \beta$ parameters. (As a matter of fact, even the exponential model is a special case with $a=1, \alpha=1, b=0$.)

Gompertz growth One of the earliest such model (used already by Laird in 1964 [5]), and perhaps the most widely used even today, is the Gompertz growth. It is governed by the following DE:

$\frac{\mathrm{d} V(t)}{\mathrm{d} t}=a V(t)-b V(t) \ln V(t)$,

giving rise to the Gompertz growth curve:

$V(t)=e^{\frac{a}{b}-\left(\frac{a}{b}-\ln V_{0}\right) e^{-b t}}$. 
(This is also the limiting case of Equation 3 when appropriate definitions are used [11].)

In the current study, the following - equivalent - parametrization will be used:

$V(t)=A e^{-b_{2} \cdot b_{3}^{t}}$,

where $A$ represent the asymptote (level of the plateau), while $b_{2}$ and $b_{3}$ determine the transition.

One well-known property of Gompertz growth is that the derivative at the initial rising period is higher than at the period before reaching the asymptote, i.e. the two transitions are not symmetric.

Logistic growth The logistic growth is another well-known sigmoid model; as opposed to the Gompertz curve, it is symmetric in both transitions.

It obeys the following DE:

$\frac{\mathrm{d} V(t)}{\mathrm{d} t}=a V(t)-b V(t)^{2}$

giving rise to the logistic growth curve:

$V(t)=\frac{a / b}{1-\left(1-\frac{a}{b V_{0}}\right) e^{-a t}}$.

(This is also a special case of Equation 3 with $\alpha=1$ and $\beta=2$.)

In the present study, the following - equivalent - parametrization will be used:

$V(t)=\frac{A}{1+e^{\frac{t_{m}-t}{s}}}$,

where $A$ represent the asymptote (level of the plateau), $t_{m}$ is the mid-point (time to reach half of the plateau level), and $s$ determines the steepness of the growth.

\section{Material and Methods}

\subsection{Experimental Setting}

\subsubsection{Mouse and Tumor Type}

Fourteen eight weeks old male C57B1/6 mice were implanted with C38 colon adenocarcinoma. A piece of tumor was transplanted subcutaneously in the recipient animal on the 1st day of the experiment. 


\subsubsection{Treatment}

We investigated the effect of bevacizumab which is an angiogenic inhibitor [12]. Two groups were created to compare the effectiveness of the prescribed, one large dose protocol and a daily, quasi-continuous treatment. The control group contained 5 mice, each one received $200 \mu \mathrm{g}$ bevacizumab dose intraperitoneally on the $-1 \mathrm{st}$ day and on the 17th day of the experiment (this bolus was designed for an 18day treatment). By contrast, mice in the case group ( 9 mice) received $1.11 \mu \mathrm{g}$ bevacizumab intraperitoneally every day from the -1st day until the last day of the experiment (the total period was 20 days); that is to say, one-tenth dose of control dose spread over 18 days.

The above dosage was selected based on the fact that the recommended administration of bevacizumab is $5-10 \mathrm{mg} / \mathrm{kg}$ dose for 2-3 weeks [13]. We have administered $10 \mathrm{mg} / \mathrm{kg}$ body weight intraperitoneally, which means $200 \mu \mathrm{g}$ bevacizumab per a mouse, since the mass of the mice in the experiment was approximately $20 \mathrm{~g}$.

\subsubsection{Tumor Volume Measurement}

Tumor volume was measured with digital caliper and small animal MRI as well.

Due to the subcutaneous localization, two dimensions of the tumor can be measured with digital caliper. The third dimension should be estimated, and assuming a certain shape, tumor volume can be approximated. Based on different estimation methods, we obtained three different tumor volume values for each measurement point [14]. Measurements with caliper were made on the 0th, 2nd, 4th, 6th, 8th, 10th, 12th, 14th, 16th, 18th and 19th days of the experiment.

In order to verify our estimation methods based on caliper measurements, we used small animal MRI for tumor volume determination as well, since MRI provides much more precise volume measurement. Measurements with small animal MRI were carried out on the 0th, 4th, 7th, 11th, 14th and 19th days of the experiment. Figure 1 shows tumor volumes using all the estimation methods.

Mice were sacrificed at the end of the treatment, in strong accordance with the 3Rs principles.

\subsection{Statistical Tools}

First, individual curves were fitted for each test animal (using each measurement method) with all three models. Fitting was performed with nonlinear least squares (NLS) $[15,16]$. Gauss-Newton algorithm turned out to be incapable of converging in every case (sigmoid growth curves were problematic, when even the end of the observed data was far from reaching the plateau). Thus, Levenberg-Marquardt algorithm [17] was employed, which successfully converged for all three models for every growth curve. 


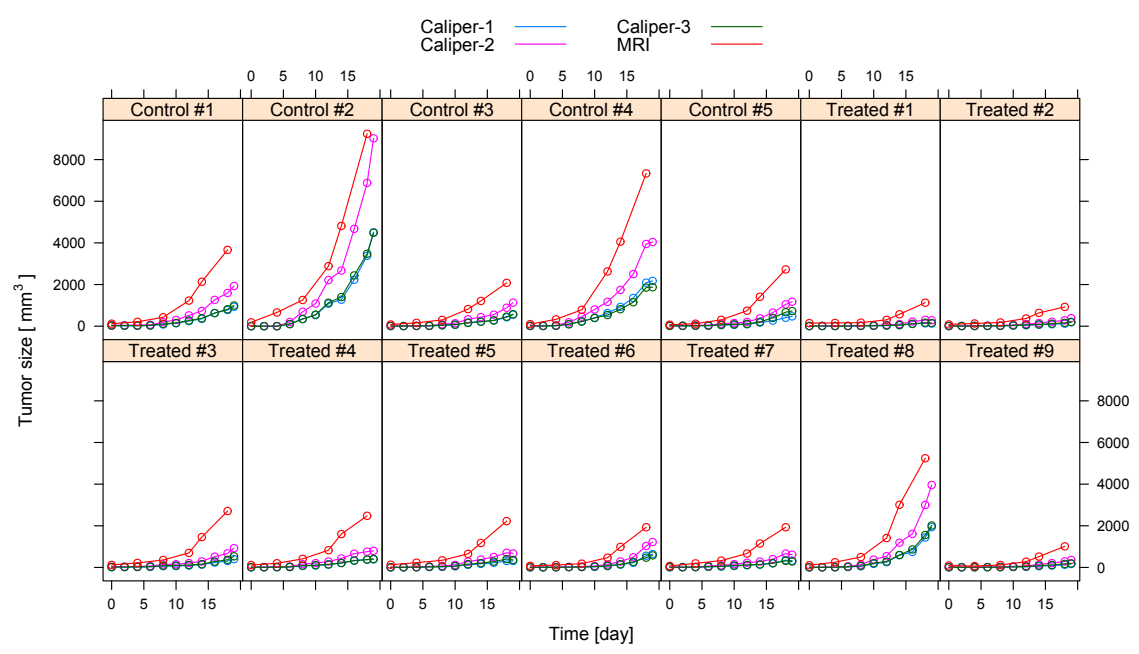

Figure 1

Measured tumor volumes (with all measurement methods).

This approach provides the best fit as it estimates individual parameters for all subject and measurement method, but this is also the very reason that prevents generalization: we have separate models, thus it is not possible to infer on a higher level (i.e. the population of parameters). As we are now primarily interested not in these particular subjects, but rather on the population from which they are coming, a model will be used which explicitly incorporates this aspect: the mixed effects model [18, 19].

These models assume that parameters are not fixed values, but rather realizations of a random variable, most typically normal random variate; this is called a random effect. These are characterized by mean and variance (and possibly covariance for different such distributions). Therefore the estimation focuses not on the individual parameters, but rather on these parameters of the population.

To formalize: denoting the tumor volume of the $i$ th subject at measurement number $j$ with $V_{i j}$ we have

$V_{i j}=f\left(\boldsymbol{\phi}_{i j}, t_{i j}\right)+\varepsilon_{i j}, \quad \varepsilon_{i j} \sim \mathscr{N}\left(0, \sigma^{2}\right)$,

where $f$ represents the nonlinear functional form - in the present study, exponential, Gompertz or logistic - of time ( $t_{i j}$ being the time when the $j$ the measurement was made on the $i$ th subject) determined the parameters $\phi_{i j}$, the dimensionality of which corresponds to the number of parameters in the growth curve ( 2 or 3 in our cases).

Now we assume that these parameters depend on whether the test animal belongs to the treated or to the control group and on the measurement method, that is

$\boldsymbol{\phi}_{i j}=\left(\boldsymbol{\beta}_{0}+\mathbf{b}_{i}\right)+\boldsymbol{\beta}_{\text {Group }} \operatorname{Group}_{i}+\boldsymbol{\beta}_{\text {MeasMeth MeasMeth }}$, 
where random effects $\mathbf{b}_{i} \sim \mathscr{N}(\mathbf{0}, \boldsymbol{\psi})$ and $\boldsymbol{\beta}$ s are vectors containing the fixed effects for each parameter of the growth curve. (In this case $\boldsymbol{\phi}_{i j}$ only depends on $i$, in other words, we had no time-varying covariates.) For instance, exponential growth curve, using the more usual notation, is modelled as

$$
\begin{aligned}
& V_{i}(t)=\left(V_{0}+b_{V_{0}, i}+\beta_{\text {Group }}^{\left(V_{0}\right)} \operatorname{Group}_{i}+\beta_{\text {MeasMeth } \left.^{\left(V_{0}\right)} \operatorname{MeasMeth}_{i}\right) .}\right.
\end{aligned}
$$

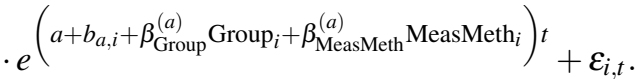

We assumed that $\boldsymbol{\psi}$ is diagonal, i.e. the random effects are uncorrelated. Residual (within-group) error $\varepsilon_{i j}$ is assumed to be independent and independent from all random effects too.

To sum up, we assume that these covariates act by altering the parameters of the same - functional form. In particular, this means that the effect of drug is incorporated by assuming that the tumor growth obeys the same law under treatment, but with different parameters.

This model assumes, among others, that

- the variance of the error terms is a constant (i.e. no heteroscedasticity present),

- the error terms are uncorrelated (i.e. no residual autocorrelation present).

These assumptions were checked by plotting the standardized residuals versus the time - the only covariate in the models - and the autocorrelation function of the residuals, respectively. In case of violation, appropriate weighting functions and withinsubject autocorrelation functions - with autoregressive, moving average (ARMA) models - were included in the models [18]. Models were characterized with Akaike's Information Criterion (AIC), among others.

This analysis updates a previous one [20] with more elaborate individual curve fitting and a more rigorous mixed-effects modelling.

\subsection{Programs Used}

$\mathrm{R}$ statistical program package [21] (version 3.3.2) was used with libraries minpack.lm [22] (version 1.2-0) and nlme [23] (version 3.1-128) to carry out the calculations using a custom script developed for this purpose that is available at the corresponding author on request. Visualizations were created with the lattice library [24] (version 0.20-34). 


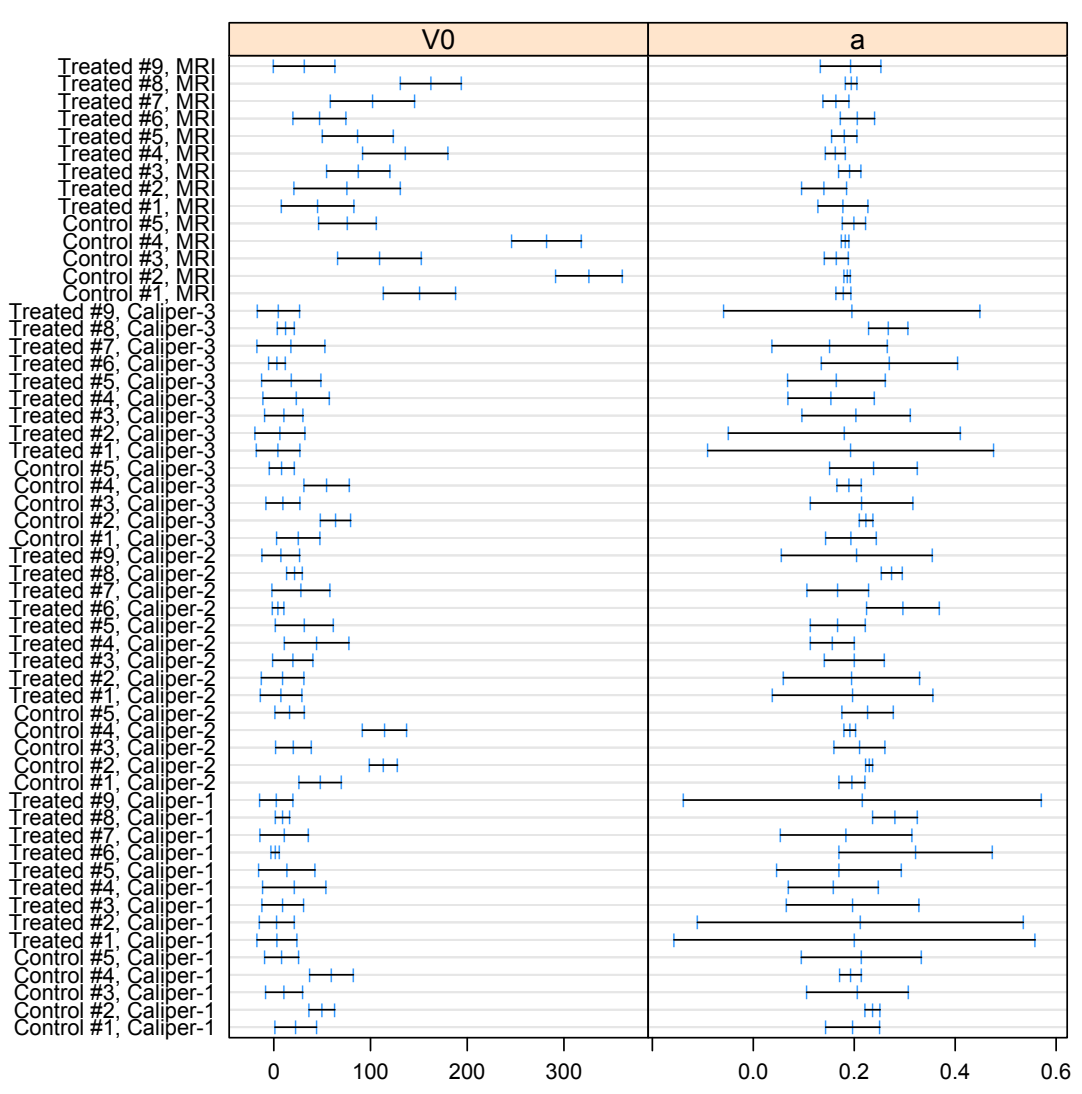

Figure 2

Point estimates with $95 \%$ confidence intervals for the parameters of the individual exponential model (for each animal using each measurement method).

\section{Results}

\subsection{Individual Fitting}

Results obtained with the exponential model are shown - for each subject and measurement method - on Figure 2.

$a$ parameters are rather homogeneous, but $V_{0}$ shows substantial heterogeneity (with a clear tendency of MRI measurements being higher than any of the caliper measurements).

The fitting of sigmoid models is much more complicated. Figure 3 shows that the parameteres associated with the plateau can be estimated only with extreme uncertainty in certain cases. (Note the logarithmic scale of the Figure.) The reason is that the data gives information only on the early phase of the growth, far from the 
plateau, which makes extrapolation very hard.

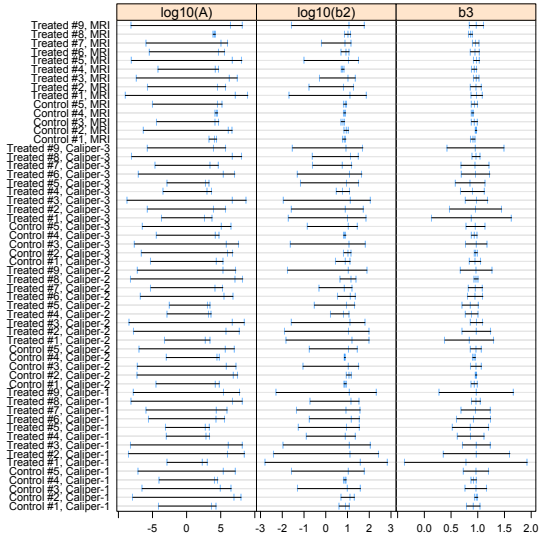

(a)

Gompertz growth

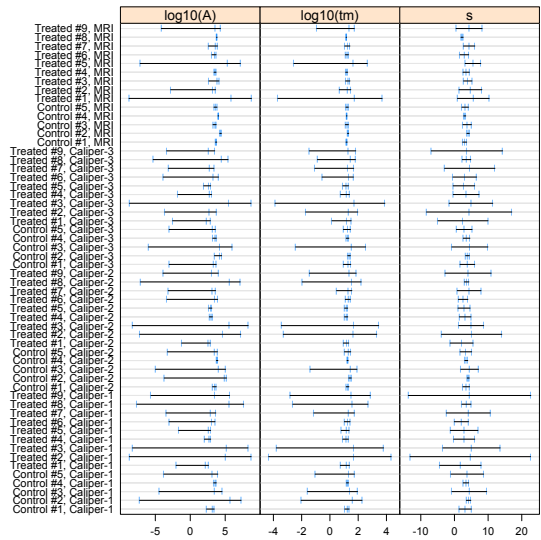

(b)

Logistic growth

Figure 3

Point estimates with $95 \%$ confidence intervals for the parameters of the individual sigmoid models (for each animal using each measurement method). Note the logarithmic scale for certain parameters.

\subsection{Population-level Mixed Model}

\subsubsection{Exponential Growth}

Residuals of the exponential model showed no substantial dependence on time, but were definitely heteroskedastic in terms of measurement method (Figure 4), so different variances were assumed for each measurement method.

The autocorrelation function (Figure 5) showed significant residual autocorrelation. As it was not disappearing even at higher lags, it was assumed to be basically MAprocess; finally ARMA $(1,4)$ specification turned out to be practically removing residual autocorrelation.

In this - now diagnostically correct - model, the standard deviation of the random effect for the $V_{0}$ was 13.14565 , for the $a$ it was 0.02614131 . The residual standard deviation and AIC is shown in Table 1.

Table 1

Residual standard deviations and AICs of different population-level models

\begin{tabular}{|c|c|c|}
\hline Model & Residual standard deviation & AIC \\
\hline Exponential & 113.2 & 6474 \\
\hline Logistic & 86.6 & 6602 \\
\hline Gompertz & 660 & 8677 \\
\hline
\end{tabular}




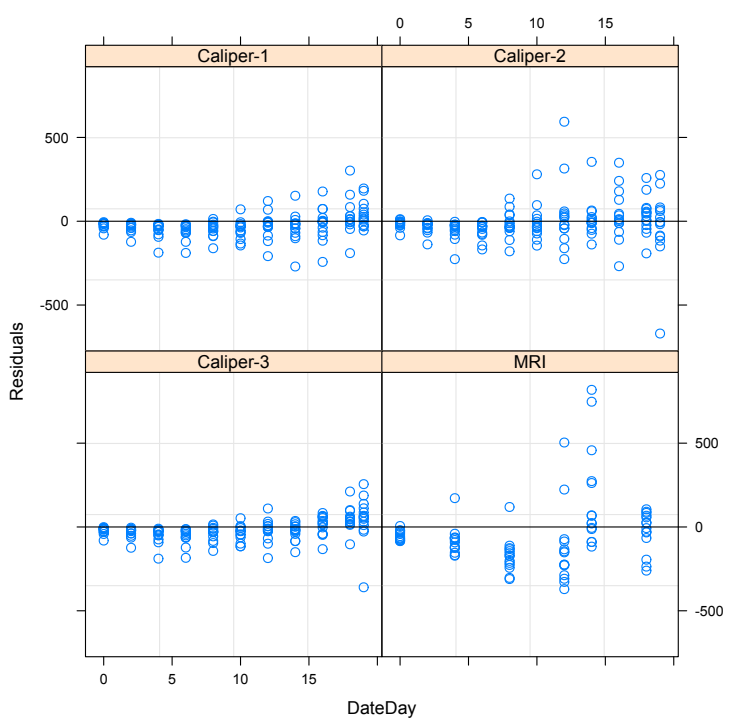

Figure 4

Residuals of the exponential model plotted as a function of day, by measurement method.

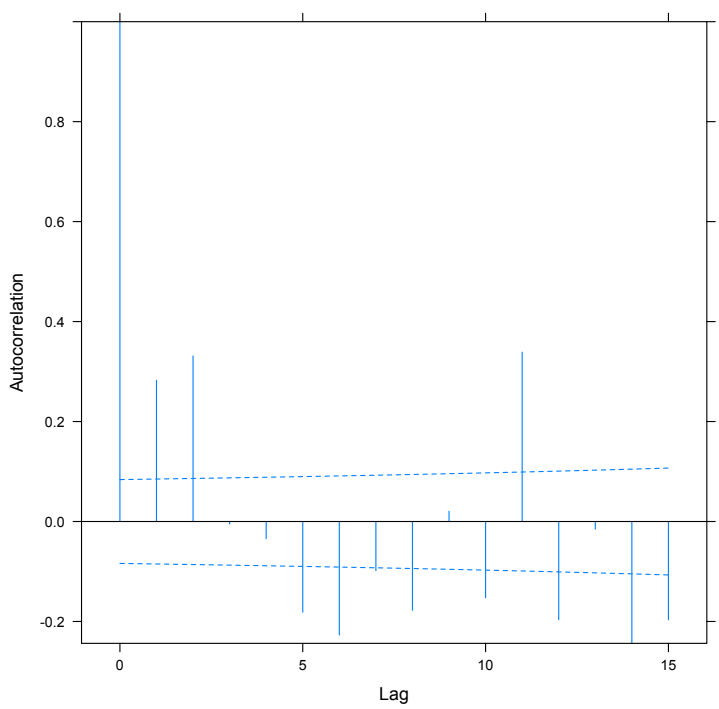

Figure 5

Autocorrelation function of the residuals from the exponential model, with critical values at $5 \%$ significance level. 


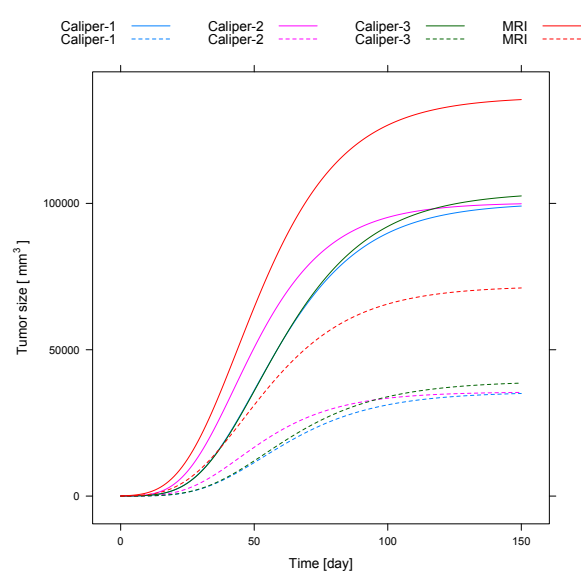

(a)

Gompertz growth

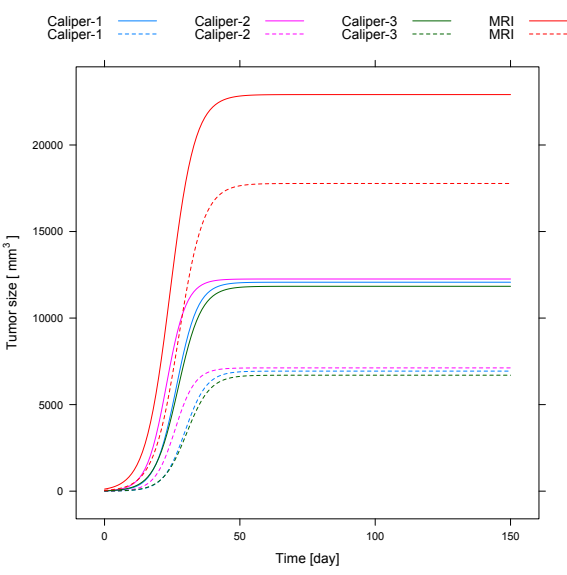

(b)

Figure 6

Predicted growth (on population-level) of the tumor volume for Days 0 to 150. Different colors indicate different measurement methods (see legend), solid line indicates control group, while dashed line indicates treated group.

In this model, the intercept of $V_{0}$ is 40.5 with MRI measurement having significantly higher values than caliper measurements (+275.2 compared to Caliper-1). The intercept of $a$ is 0.18349 , but now MRI measurements are having significantly lower $a$ $(-0.04342$ compared to Caliper-1), Caliper-2 however exhibits significantly higher $a(+0.02894)$.

The treatment's effect is very interesting: it does slightly significantly alter $V_{0}$ $(-18.05230, p=0.0354)$, but it does have a more significant effect on $a$, being associated with -0.03746 change $(p=0.0197)$. I.e. the continuous regimen decreases the rate of growth - which shows the benefit of this dosing regimen.

\subsubsection{Sigmoid Growth}

Sigmoid growth models were barely approximable (as already expected from the results of the individual fitting), so no attempt was made to include special variance or autocorrelation function.

The results of the mixed models are shown (Figure 6) as - population-level - predicted tumor volumes for Days 0 to 150 (for each measurement type and for the treated/control groups). Note that the original data spanned from Days 0 to 19 , so this exemplifies the extrapolation with the models.

The residual standard deviations and AICs are shown in Table 1.

The effect of treatment was significant for the mid-point parameter of the logistic growth ( $p=0.0228)$, but it was not significant for any parameter of the Gompertz growth. In particular, the asymptotic levels were substantially lower in both cases 
( -5136 with the intercept being 12071 for the logistic model, -64657 with the intercept being 100263 for the Gompertzian model), but these were insignificant, as they were very hard to estimate, as expected (also note the extreme difference between the two models in asymptotic levels).

The asymptotic level in the logistic model was significantly - and substantially higher with MRI $(+10841, p<0.001)$.

\section{Discussion}

It was possible to reliably estimate all parameters of the exponential growth model, indicated by the consistent and rather narrow confidence intervals in the individual fits and the acceptable residual standard deviation in the mixed model. The results show the effect of the treatment; already demonstrated in an earlier research [25].

In contrast, the estimates for the sigmoid-growth models were exceedingly variable evidenced by the very wide confidence intervals. The population level model exhibited extremely poor fit, with enormously high residual deviations. Nevertheless, it could concluded that the logistic modell still provided better fit than the Gompertzian.

In short, it was not possible to reliably estimate these models, the reason being the rather short observation period that was available, showing only the very early period of tumor growth. Notwithstanding, results point out the possibility to estimate the plateu phase, which is a very interesting and promosing parameter - in additional to the initial rate of growth, already captured by the exponential model -, but trusthworthy estimation of this requires more observation.

Results also highlight the differences between the measurement devices, clearly showing that measurements made with MRI are systematically higher than any of the caliper measurements.

One strength of our approach was that it integrates all factors (treatment and measurement device) into one single model, allowing us to investigate both the effect of the treatment and the effect of the applied measuring method at the same time. Also, the mixed effects approach allows a smooth and elegant usage of the individual measurements to create a population-level model. In addition to that, we have the possibility to test several potentional functional forms; with some of them, we are also able to extrapolate in time, and capture clinically relevant parameters.

The most important limitation was the rather small number of test subjects, and the too short observation period to estimates some of the models.

\section{Conclusion}

The exponential model could be estimated in a robust manner, both individually, and in the population-level with the mixed effects model. Results confirm the effect of the treatment, and make it possible to quantify this. 
In contrast, the sigmoid-like growth curves were almost impossible to estimate, revealing the limitations of our data. Nevertheless, the possibility to estimate such models - making extrapolation possible - presents a promising opportunity.

\section{Acknowledgement}

This project has received funding from the European Research Council (ERC) under the European Union's Horizon 2020 research and innovation programme (grant agreement No. 679681). In addition, Tamás Ferenci was supported by the UNKP16-4/III New National Excellence Program of the Ministry of Human Capacities. The authors say thanks to the Physiological Controls Group's (http://physcon . uni-obuda.hu/) members for their support.

\section{References}

[1] R. M. A. Parker and W. J. Browne. "The Place of Experimental Design and Statistics in the 3Rs". In: ILAR Journal 55.3 (2014), pp. 477-485.

[2] I. Petersen. "Antiangiogenesis, anti-VEGF(R) and outlook". In: Recent Results In Cancer Research, Targeted Therapies in Cancer. Ed. by M. Dietel. Springer - Verlag, 2007.

[3] G. Bergers and L. E. Benjamin. "Tumorigenesis and the angiogenic switch". In: Nat Rev Cancer. 3(6) (2003), pp. 401-410.

[4] P. Hahnfeldt et al. "Tumor development under angiogenic signaling: A dynamical theory of tumor growth, treatment response, and postvascular dormancy". In: Cancer research 59 (1999), pp. 4770-4775.

[5] A. K. Laird. "Dynamics of tumour growth". In: British Journal of Cancer 18.3 (1964), p. 490.

[6] M. Simeoni et al. "Predictive pharmacokinetic-pharmacodynamic modeling of tumor growth kinetics in xenograft models after administration of anticancer agents". In: Cancer Research 64.3 (2004), pp. 1094-1101.

[7] S. Yamazaki et al. "Pharmacokinetic-pharmacodynamic modeling of biomarker response and tumor growth inhibition to an orally available cMet kinase inhibitor in human tumor xenograft mouse models". In: Drug Metabolism and Disposition 36.7 (2008), pp. 1267-1274.

[8] P. L. Bonate. "Modeling tumor growth in oncology". In: Pharmacokinetics in drug development. Springer, 2011, pp. 1-19.

[9] R. P. Araujo and D. L. S. McElwain. "A history of the study of solid tumour growth: the contribution of mathematical modelling". In: Bulletin of mathematical biology 66.5 (2004), pp. 1039-1091.

[10] S. Benzekry et al. "Classical mathematical models for description and prediction of experimental tumor growth". In: PLoS Comput Biol 10.8 (2014), e1003800.

[11] M. Marušić et al. "Analysis of growth of multicellular tumour spheroids by mathematical models". In: Cell proliferation 27.2 (1994), pp. 73-94. 
[12] European Medicines Agency. Scientific discussion of Avastin. Available: http: //www. ema. europa. eu. Accessed 10 April 2016. 2005.

[13] Genentech. Prescribing information of Avastin (Bevacizumab). Available: http: //www . gene. com/download/pdf/avastin_prescribing.pdf. Accessed 10 December 2016. 2013.

[14] J. Sápi et al. "Tumor Volume Estimation and Quasi-Continuous Administration for Most Effective Bevacizumab Therapy”. In: PLoS ONE 10.11 (Nov. 2015), pp. 1-20. DOI: 10.1371/journal . pone.0142190.

[15] D. M. Bates and D. G. Watts. Nonlinear regression analysis and lts applications. Wiley, 1988.

[16] C. Ritz and J. Streibig. Nonlinear Regression with R. Use R! Springer New York, 2008. ISBN: 9780387096162.

[17] J. J. Moré. "The Levenberg-Marquardt algorithm: Implementation and theory". In: Numerical Analysis: Proceedings of the Biennial Conference Held at Dundee, June 28-July 1, 1977. Ed. by G. A. Watson. Berlin, Heidelberg: Springer Berlin Heidelberg, 1978, pp. 105-116. ISBN: 978-3-540-35972-2. DOI: 10 . 1007 / BFb0067700. URL: http : / / link . springer . com / chapter/10.1007\%2FBFb0067700.

[18] J. Pinheiro and D. Bates. Mixed-Effects Models in S and S-PLUS. Statistics and Computing. Springer New York, 2000. ISBN: 9780387989570.

[19] G. Verbeke and G. Molenberghs. Linear Mixed Models for Longitudinal Data. Springer Series in Statistics. Springer New York, 2009. ISBN: 9781441902993.

[20] T. Ferenci, J. Sápi, and L. Kovács. "Modelling xenograft tumor growth under antiangiogenic inhibitation with mixed-effects models". In: Systems, Man, and Cybernetics (SMC), 2016 IEEE International Conference on. 2016, pp. 3912 3917.

[21] R Core Team. R: A Language and Environment for Statistical Computing. R Foundation for Statistical Computing. Vienna, Austria, 2016. URL: https : //www.R-project.org/.

[22] T. V. Elzhov et al. minpack.lm: R Interface to the Levenberg-Marquardt Nonlinear Least-Squares Algorithm Found in MINPACK, Plus Support for Bounds. R package version 1.2-0. 2015. URL: https : / CRAN . R-project . org/ package $=$ minpack. $1 \mathrm{~m}$.

[23] J. Pinheiro et al. nlme: Linear and Nonlinear Mixed Effects Models. R package version 3.1-128. 2016. URL: http : / CRAN . R-project . org / package= nlme.

[24] D. Sarkar. Lattice: Multivariate Data Visualization with R. ISBN 978-0-38775968-5. New York: Springer, 2008. URL: http: //Imdvr.r-forge.rproject.org.

[25] J. Sápi et al. "Tumor Model Identification and Statistical Analysis". In: Systems, Man, and Cybernetics (SMC), 2015 IEEE International Conference on. 2015, pp. 2481-2486. DOI: 10.1109/SMC. 2015. 434. 